Document downloaded from:

http://hdl.handle.net/10251/78363

This paper must be cited as:

Ruiz Rico, M.; Daubenschüz, H.; Pérez Esteve, E.; Marcos Martínez, MD.; Amorós, P.; Martínez Mañez, R.; Barat Baviera, JM. (2016). Protective effect of mesoporous silica particles on encapsulated folates. European Journal of Pharmaceutics and Biopharmaceutics. 105:9-17. doi:10.1016/j.ejpb.2016.05.016.

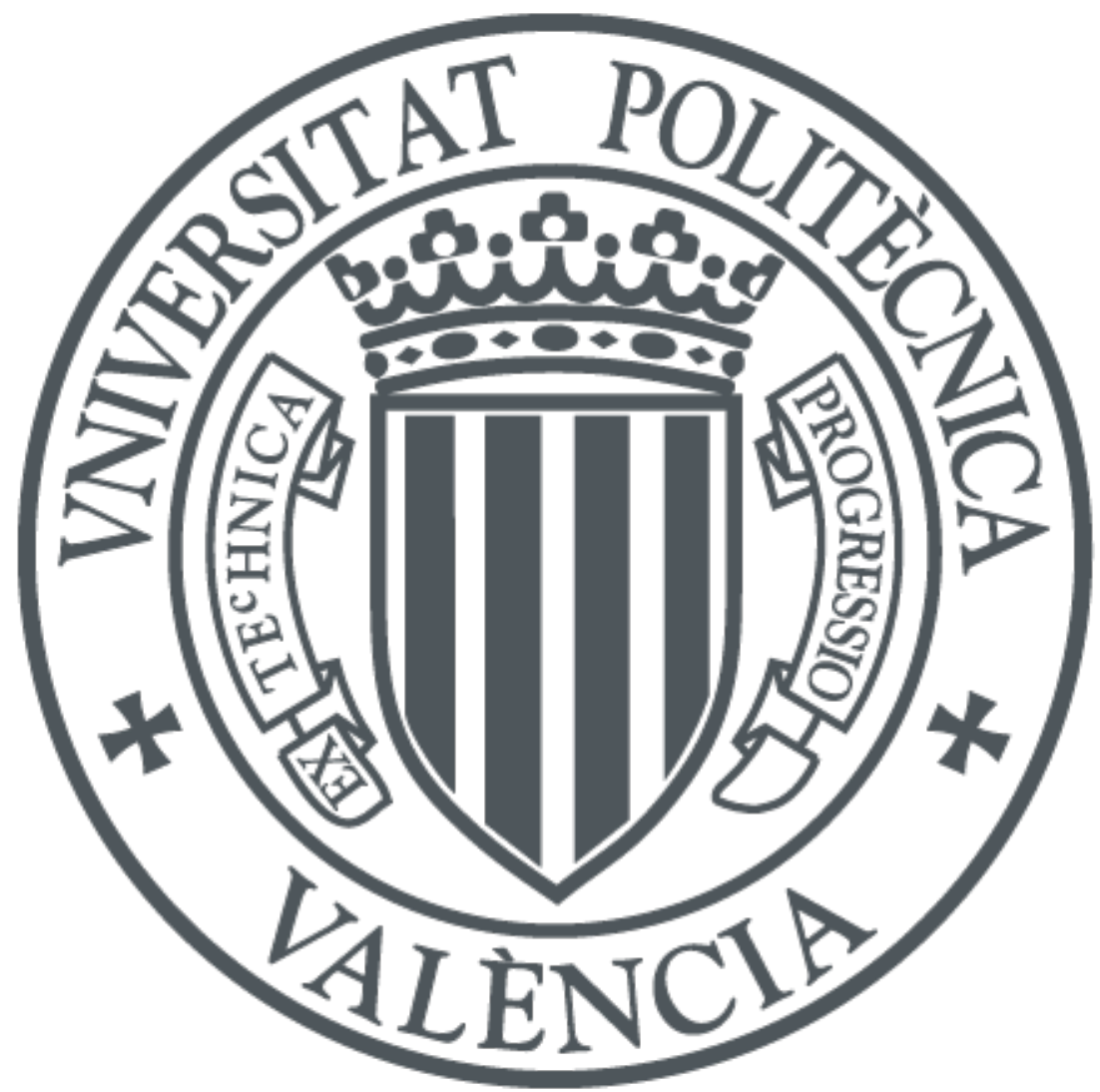

The final publication is available at

http://dx.doi.org/ 10.1016/j.ejpb.2016.05.016

Copyright Elsevier

Additional Information 


\section{Protective effect of mesoporous silica particles on encapsulated folates}

2

3 María Ruiz-Rico ${ }^{1, *}$, Hanna Daubenschüz ${ }^{1}$, Édgar Pérez-Esteve ${ }^{1}$, María D. Marcos ${ }^{2,3}$, Pedro

4 Amorós ${ }^{4}$, Ramón Martínez-Máñez ${ }^{2,3}$, José M. Barat ${ }^{1}$

5

$6{ }^{1}$ Grupo de Investigación e Innovación Alimentaria. Departamento de Tecnología de Alimentos,

7 Universitat Politècnica de València. Camino de Vera s/n, 46022, Valencia, Spain

$8{ }^{2}$ Centro de Reconocimiento Molecular y Desarrollo Tecnológico (IDM), Unidad Mixta

9 Universitat Politècnica de València y Universitat de València. Departamento de Química,

10 Universitat Politècnica de València, Camino de Vera s/n, 46022, Valencia, Spain

$11{ }^{3}$ CIBER de Bioingeniería, Biomateriales y Nanomedicina (CIBER-BBN)

$12{ }^{4}$ Institut de Ciència dels Materials (ICMUV), Universitat de València, P.O. Box 22085, 46071

13 Valencia, Spain

14 *(M.R.R.) Phone: +34963877000 ext.83612. E-mail: maruiri@etsia.upv.es. 
Mesoporous silica particles (MSPs) are considered suitable supports to design gated materials for the encapsulation of bioactive molecules. Folates are essential micronutrients which are sensitive to external agents that provoke nutritional deficiencies. Folates encapsulation in MSPs to prevent degradation and to allow their controlled delivery is a promising strategy. Nevertheless, no information exists about the protective effect of MSPs encapsulation to prevent their degradation. In this work, 5-formyltetrahydrofolate (FO) and folic acid (FA) were entrapped in MSPs functionalized with polyamines, which acted as $\mathrm{pH}$-dependent molecular gates. The stability of free and entrapped vitamins after acidic $\mathrm{pH}$, high temperature and light exposure was studied. The results showed the degradation of FO after high temperature and acidic $\mathrm{pH}$, whereas entrapped FO displayed enhanced stability. Free FA was degraded by light, but MSPs stabilized the vitamin. The obtained results point towards the potential use of MSPs as candidates to enhance stability and to improve the bioavailability of functional biomolecules.

Keywords: 5-formyltetrahydrofolate; controlled release; encapsulation; folic acid; mesoporous silica particles; stability

\section{Abbreviations used} 5-formyltetrahydrofolate (FO), ascorbic acid (AA), encapsulated 5-formyltetrahydrofolate (EFO), encapsulated folic acid (E-FA), European Food Safety Authority (EFSA), folic acid (FA), free 5-formyltetrahydrofolate (F-FO), free folic acid (F-FA), least significant difference (LSD), mesoporous silica particles (MSPs), N-(3-trimethoxysilylpropyl)diethylenetriamine (N3), Ncetyltrimethylammonium bromide (CTABr), phosphate-buffered saline (PBS), powder X-ray diffraction (PXRD), tetrabutylammonium hydrogen sulphate (TBAHS), tetraethylorthosilicate (TEOS), transmission electron microscopy (TEM), triethanolamine ( $\left.\mathrm{TEAH}_{3}\right)$, ultraviolet (UV). 


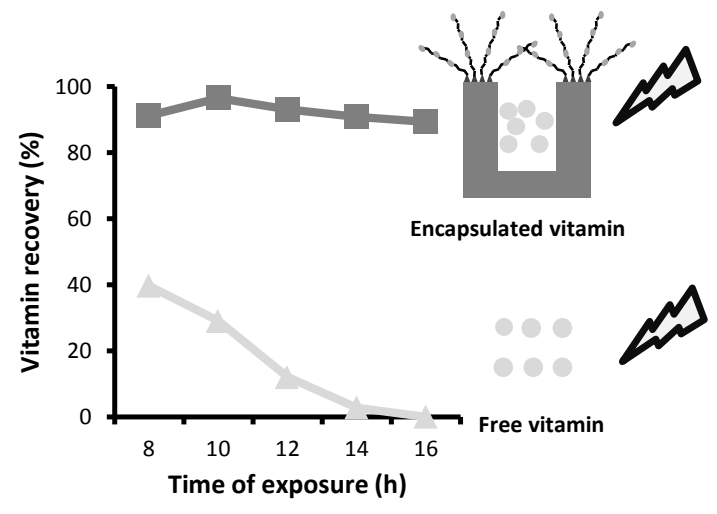


In the last years hybrid organic-inorganic materials have attracted considerable interest due to the combination of the beneficial characteristic of organic chemistry and material science in order to develop smart nanodevices. Among different hybrid solids, mesoporous silica particles (MSPs) offer several unique features that allow the design of gated materials for controlled release and sensing/recognition protocols [1]. The first family of MSPs called MCM-X was described in the early 1990s by the Mobil Corporation Laboratories. This family of silica supports include different porous silica exhibiting hexagonal (MCM-41), cubic (MCM-48) and lamellar (MCM-50) pore shapes. After these developments, new ordered materials (e. i. MSU, KIT, FDU, AMS, SBA...) with a wide range of textural properties have been described by different authors [2]. MSPs possess broadly advantageous properties such as biocompatibility, thermal and chemical stability, huge loading capacity, high surface areas, tunable morphologies and pore sizes, as well as facile functionalization of surfaces and pores [3-7]. The surface functionalization of MSPs for the development of gated materials allows that the delivery of the cargo stored in the inorganic support can be triggered by applying selected external stimulus [1]. Furthermore, it is considered that the inorganic framework can effectively protect the payload molecules from enzymatic degradation or denaturation caused by environmental changes [7]. However, there are few studies in the literature about the protective effect of MSPs on the stability of biomolecules in biological solutions.

The functionalized MSPs have been used to encapsulate drugs mainly for the biomedical field, but also to encapsulate bioactive molecules for other sectors such as food technology. Different food ingredients and nutraceuticals including vitamins [8-12], antioxidants [13,14], antimicrobials [15-17], aromas [18] or enzymes [19] have been entrapped in gated mesoporous materials. Most studies have been focused on the development and optimization of the encapsulation systems for controlled delivery, but it is expected that the MPSs may be able to enhance the stability of the entrapped bioactive compound. 
Water-soluble vitamins, like folates, are labile compounds in the presence of environmental agents, such as extreme $\mathrm{pH}$ values or high temperatures [20]. As folates are essential for the human body and cannot be synthesized de novo by the organism, this indispensable vitamin needs to be obtained from food or dietary supplements [21]. Thus the stability of this vitamin after storage and processing in food products or supplements should be taken into account. Loss of the biochemical activity of natural folates can occur during harvest, storage and food processing $[22,23]$. In general, $\mathrm{pH}$, temperature, pressure, light and antioxidants, among others, can affect the stability of the natural folates and the synthetic folic acid (FA) [24-30]. FA, with a fully oxidized pteridine ring system, exhibits greater stability than folates. Among folates, large differences in stability exist in susceptibility to oxidative degradation, and 5formyltetrahydrofolate (FO) is the most stable [31]. Moreover, the stability of folates is influenced by $\mathrm{pH}$ and oxygen, which provokes their oxidation [30,32]. The inclusion of antioxidant compounds, such as ascorbic acid (AA) or mercaptoethanol, is required to prevent

83 the destruction of labile folates from thermal exposure and photodegradation during food 84 processing $[20,23,33]$.

Bearing in mind these factors, it is of interest to create folates encapsulation systems which can ensure the required dose and fully guarantee the stability and bioavailability of this vitamin. Therefore, the objective of this study was the encapsulation of FO and FA in a mesoporous silica support (MCM-41) functionalized with amines to create a system to be used in orally delivered applications, and to study the stability of entrapped vitamins to test the efficacy of the MCM-41 support as a protector against external agents, such as acidic $\mathrm{pH}$, high temperature and

91 light. 
94

\section{Chemicals}

Tetraethylorthosilicate (TEOS), $N$-cetyltrimethylammonium bromide (CTABr), sodium hydroxide $(\mathrm{NaOH})$, triethanolamine $\left(\mathrm{TEAH}_{3}\right), N$-(3-trimethoxysilylpropyl)diethylenetriamine (N3), sodium phosphate monobasic $\left(\mathrm{NaH}_{2} \mathrm{PO}_{4}\right)$, sodium phosphate dibasic $\left(\mathrm{Na}_{2} \mathrm{HPO}_{4}\right)$ and tetrabutylammonium hydrogen sulphate (TBAHS) were provided by Sigma-Aldrich (Madrid, Spain). 5-formyltetrahydrofolate (FO) and folic acid (FA) were purchased from Schircks Laboratories (Jona, Switzerland). Acetonitrile HPLC grade was provided by Scharlab (Barcelona, Spain).

\section{Mesoporous silica particles synthesis}

Synthesis of microparticulated MCM-41 particles was carried out following the so-called "atrane route", where CTABr was used as the structure-directing agent. A molar ratio, fixed at 7 TEAH ${ }_{3}: 2$ TEOS:0.52 CTABr:0.5 NaOH: $180 \mathrm{H}_{2} \mathrm{O} . \mathrm{CTABr}$, was added to a TEAH $\mathrm{H}_{3}$ and $\mathrm{NaOH}$ solution, which contained TEOS at $118{ }^{\circ} \mathrm{C}$. After dissolving CTABr in the solution, water was slowly added along with vigorous stirring at $70{ }^{\circ} \mathrm{C}$ to form a white suspension. This mixture was aged at $100{ }^{\circ} \mathrm{C}$ for $24 \mathrm{~h}$. Following synthesis, the solid was recovered, washed with deionized water and dried at $70{ }^{\circ} \mathrm{C}$. The as-synthesized microparticles were calcined at $550{ }^{\circ} \mathrm{C}$ in an oxidant atmosphere for $5 \mathrm{~h}$ to remove the template phase [16].

\section{Synthesis of encapsulated folates}

The design of the encapsulation system was based on a previous work, in which FA was entrapped in a MSP functionalized with amines to deliver FA during a simulated digestion process [12]. Dissolutions of FO and FA $(10 \mathrm{mg} / \mathrm{mL})$ were prepared in distilled water and phosphate-buffered saline (PBS), respectively. Solutions were added to $300 \mathrm{mg}$ of MCM-41 in 3 addition cycles $\left(1.5 \mathrm{~mL}\right.$ per cycle). After each addition cycle, solids were dried at $37{ }^{\circ} \mathrm{C}$ to 
remove water content. After loading and drying, solids were collected and functionalized with $1.29 \mathrm{~mL}$ of $\mathrm{N} 3 \mathrm{using}$ different media; i.e. acetonitrile (E-FO) or acetate buffer at $\mathrm{pH} 2$ (E-FA). The final mixtures were stirred for $5.5 \mathrm{~h}$ at room temperature, isolated by vacuum filtration, washed with $300 \mathrm{~mL}$ of water adjusted to $\mathrm{pH} 2$, and dried at room temperature for $24 \mathrm{~h}$.

\section{Characterization of solids}

Powder X-ray diffraction (PXRD), transmission electron microscopy (TEM), $\mathrm{N}_{2}$ adsorptiondesorption isotherms and zeta potential were used to characterize the synthesized materials. PXRD was performed in a BrukerD8 Advance diffractometer using $\mathrm{CuK} \alpha$ radiation (Bruker, Coventry, UK). For the TEM analysis, particles were dispersed in dichloromethane and sonicated for 2 min to preclude aggregates. The suspension was then deposited onto copper grids coated with a carbon film (Aname SL, Madrid, Spain). The MSPs samples were imaged by JEOL JEM-1010 (JEOL Europe SAS, Croissy-sur-Seine, France) at an acceleration voltage of $80 \mathrm{kV}$. The single-particle size was estimated by averaging the measured size values of 50 particles. The $\mathrm{N}_{2}$ adsorption-desorption isotherms were recorded with a Micrometrics ASAP2010 automated sorption analyzer (Micromeritics Instrument Corporation, Norcross, USA). Samples were degassed at $90{ }^{\circ} \mathrm{C}$ in vacuum overnight. Specific surface areas were calculated from the adsorption data within the low pressure range by the BET model. Pore size was determined following the $\mathrm{BJH}$ method. To determine the zeta potential of the materials, a Zetasizer Nano ZS (Malvern Instruments, UK) was employed. Samples were dispersed in water at a concentration of $1 \mathrm{mg} / \mathrm{mL}$. Before taking each measurement, samples were sonicated for 2 min to preclude aggregation. The zeta potential was calculated from the particle mobility values by applying the Smoluchowski model. The average of five recordings was reported as the zeta potential. Measurements were taken at $25^{\circ} \mathrm{C}$ in triplicate. 
145 Delivery studies were conducted to test the release capacity of the encapsulation system and to

146 confirm the functionality of the gates to modulate the release of vitamins according to the $\mathrm{pH}$ of 147 the medium (closed gates at $\mathrm{pH} 2$, opened gates at $\mathrm{pH} 7.5$ ). To determine the release of $\mathrm{FO}$ and 148 FA from the amine-gated mesoporous support (E-FO and E-FA), $10 \mathrm{mg}$ of the solids were 149 placed in $25 \mathrm{~mL}$ of PBS at $\mathrm{pH} 2$ and $\mathrm{pH}$ 7.5. At certain time points $(0,2,5,15,30,60,120,180$ min), aliquots were separated, the suspension was filtered and the solution was analyzed by HPLC.

\section{Stability assays}

154 The influence of diverse external agents, such as acidic $\mathrm{pH}$, high temperature and light, on the 155 stability of the free and entrapped vitamin was studied. Free FO and FA were treated, whenever possible, as the encapsulated vitamin in order to ensure reproducibility. In order to simulate not only the 3 day-loading $\left(72 \mathrm{~h}\right.$ drying at $\left.37^{\circ} \mathrm{C}\right)$ of the particles with the vitamin, but also a further 24-hour drying period after functionalization, compounds in their free form were incubated for $96 \mathrm{~h}$ at $37^{\circ} \mathrm{C}$. The stability assays with the free vitamin were all conducted with these incubated samples (F-FO and F-FA).

For the stability assays, $4 \mathrm{mg}$ of the entrapped vitamins (E-FO and E-FA) and the correspondent amounts of the free forms (ca. $0.02 \mathrm{mg}$ for FO and ca. $0.3 \mathrm{mg}$ for FA) were dissolved in $10 \mathrm{~mL}$ of PBS ( $\mathrm{pH} 2$ or $\mathrm{pH} 7.5$ ). All the stability experiments were performed in triplicate. The vitamin recoveries were presented by assuming the percentage recovered under optimal conditions to be $100 \%$ (pH 7.5, no treatment).

pH stability

These experiments were carried out to study the stability and solubility of vitamins at different pH values; e.g., the acidic $\mathrm{pH}$ at which FO and FA exhibited very low solubility [30,32]. These 
assays allow us to confirm the mechanism of polyamines as molecular gates due to the transformation of amines (open gate at a neutral/basic $\mathrm{pH}$ ) into polyammonium groups (closed gate at an acidic $\mathrm{pH})$.

Firstly, the recovery of free compounds under different $\mathrm{pH}$ conditions $(\mathrm{pH} \mathrm{1,} \mathrm{2,} \mathrm{3,} \mathrm{4,} \mathrm{5,} \mathrm{6,} \mathrm{7,} \mathrm{8,}$ 9, 10) was examined. Depending on the $\mathrm{pH}$ value, $\mathrm{PBS}$ was adjusted with $\mathrm{H}_{2} \mathrm{SO}_{4}$ and $\mathrm{NaOH} 1$ $\mathrm{M}$ according to $\mathrm{Wu}$ et al. [32]. Then the correspondent volume of a stock solution of vitamins was added. After stirring samples for $1 \mathrm{~h}$, they were taken for the HPLC analysis. The second part of the $\mathrm{pH}$ assays was conducted to test the stability behavior of vitamins after neutralization and to prove the functionality of ascorbic acid and the $\mathrm{pH}$-responsive gated support to protect vitamins. The encapsulated vitamins were mixed with PBS (pH 2), stirred for $1 \mathrm{~h}$ and samples were taken. $\mathrm{pH}$ was adjusted to neutral $\mathrm{pH}$ with $\mathrm{NaOH} 5 \mathrm{M}$ and vitamins were released. The same procedure was carried out with the free forms in the presence or absence of ascorbic acid.

\section{Temperature stability}

Temperature experiments were performed in an autoclave. Free vitamins were dissolved in PBS (pH 7.5) and equivalent amounts of encapsulated vitamin were suspended in PBS (pH 2) to keep the gates closed, and to then undergo the sterilization process. Treatment was conducted at $121^{\circ} \mathrm{C}$ and 1 bar at different times: 5, 10, 15 min. After treatment, vessels were cooled in an ice bath before taking samples to be analyzed. The encapsulated samples were released by adjusting the $\mathrm{pH}$ of the suspension from $\mathrm{pH} 2$ to a neutral $\mathrm{pH}$. Delivery was done as previously described.

\section{Light stability}

Two different light sources (visible and ultraviolet (UV) lamps) were used in the stability assays. Samples were prepared in their free forms (dissolved in PBS pH 7.5) and the impact of ascorbic acid as an antioxidant (0.1\% AA dissolved in PBS) was partially examined. Experiments were conducted on encapsulated vitamins in PBS ( $\mathrm{pH}$ 2) and were adjusted to $\mathrm{pH}$ 
7.5 after the experiments, as reported above. All the samples were kept inside closed transparent borosilicate glass vessels ( $\varnothing 24 \mathrm{~mm}, \mathrm{~h} 45 \mathrm{~mm}$ ) for different times in order to simulate an indirect light-induced stress, which can actually occur in real food products. Release of vitamins was conducted as explained above.

\section{Folate and folic acid quantification}

FO and FA were determined by reversed-phase HPLC following the method described by Pérez-Esteve et al. [12]. The HPLC instrument consisted in a Hitachi LaChrom Elite liquid chromatograph (Hitachi Ltd., Tokyo, Japan), equipped with an auto-sampler (modul L-2200) and an UV detector (model L-2400). A Kromaphase $100 \mathrm{C} 18$ (250 mm x $4.6 \mathrm{~mm}$ i.d., $5 \mu \mathrm{m}$ particle size analytical column) (Scharlab, Barcelona, Spain) was used for separations. The wavelength of the UV detector was set at $280 \mathrm{~nm}$. The mobile phase consisted in (A) $0.125 \mathrm{mM}$ of $\mathrm{NaH}_{2} \mathrm{PO}_{4}, 0.875 \mathrm{mM}$ of $\mathrm{Na}_{2} \mathrm{HPO}_{4}$ and $0.4 \mathrm{mM}$ of TBAHS in water and (B) an acetonitrilemobile phase A 65:35 (v/v). The gradient program was: the mobile phase was run isocratically for the first 5 min with $90 \% \mathrm{~A}$ and $10 \% \mathrm{~B}$. The percentage of B was linearly increased to reach $36 \%$ at $15 \mathrm{~min}$ and $60 \%$ at $30 \mathrm{~min}$. The percentage of B was lowered linearly to the original composition in $5 \mathrm{~min}$, and remained under the initial conditions for $5 \mathrm{~min}$. FO and FA were quantified according to the external standard method, in which a calibration curve of the peak area was used against the compound concentration.

\section{Data analysis}

Statistical data processing was performed using Statgraphics Centurion XVI (Statpoint Technologies, Inc., Warrenton, VA, USA). The influence of the different factors on the release and stability of the vitamin was analyzed by one-way analysis of variance (One-way ANOVA). The LSD procedure (least significant difference) was used to test for the differences between means at the $5 \%$ significance level. 


\section{Results and discussion}

\section{Synthesis and material characterization}

225 FO and FA were encapsulated in the MSPs that contained gate-like ensembles as devices for 226 controlled delivery applications. In this work, diethylenetriamine moiety was chosen as the 227 capping ensemble given its proven properties to control the delivery of cargo molecules from 228 the voids of MSPs in response to changes in $\mathrm{pH}[8,12,34]$. In a first step, the support was synthesized by using CTABr as a structure director agent and TEOS as a silica source. After removing the surfactant by calcination the starting MCM-41 support was obtained. The pores of MCM-41 were loaded with FO and FA. To obtain the final materials (E-FO and E-FA), the loaded solids were reacted with $N$-(3-trimethoxysilylpropyl)diethylenetriamine.

The different supports were characterized by standard techniques. The X-ray patterns of solids MCM-41 as synthesized (a), calcined (b), loaded with FO and functionalized with amines (c) and loaded with FA and functionalized with amines (d) can be found in Figure 1. Curve a shows the expected four peaks of a hexagonal ordered array indexed as (100), (110), (200) and (210) Bragg reflections. A significant shift in the (100) reflection in the PXRD spectrum of the MCM41 calcined sample is clearly seen on curve b, which corresponds to a cell contraction related to the condensation of silanols in the calcination step. Curves $\mathrm{c}$ and d show that reflections (110),

240 (200) and (210) were lost, probably due to a reduced contrast, which can be attributed to the 241 presence of FO or FA in the pores, and to the anchored N3 molecule. Nevertheless, the existence of the (100) peak in the PXRD patterns in all cases indicated that the process of pore loading with FO and FA, and functionalization did not basically modify the typical porosity of the mesoporous MCM-41 scaffold. 


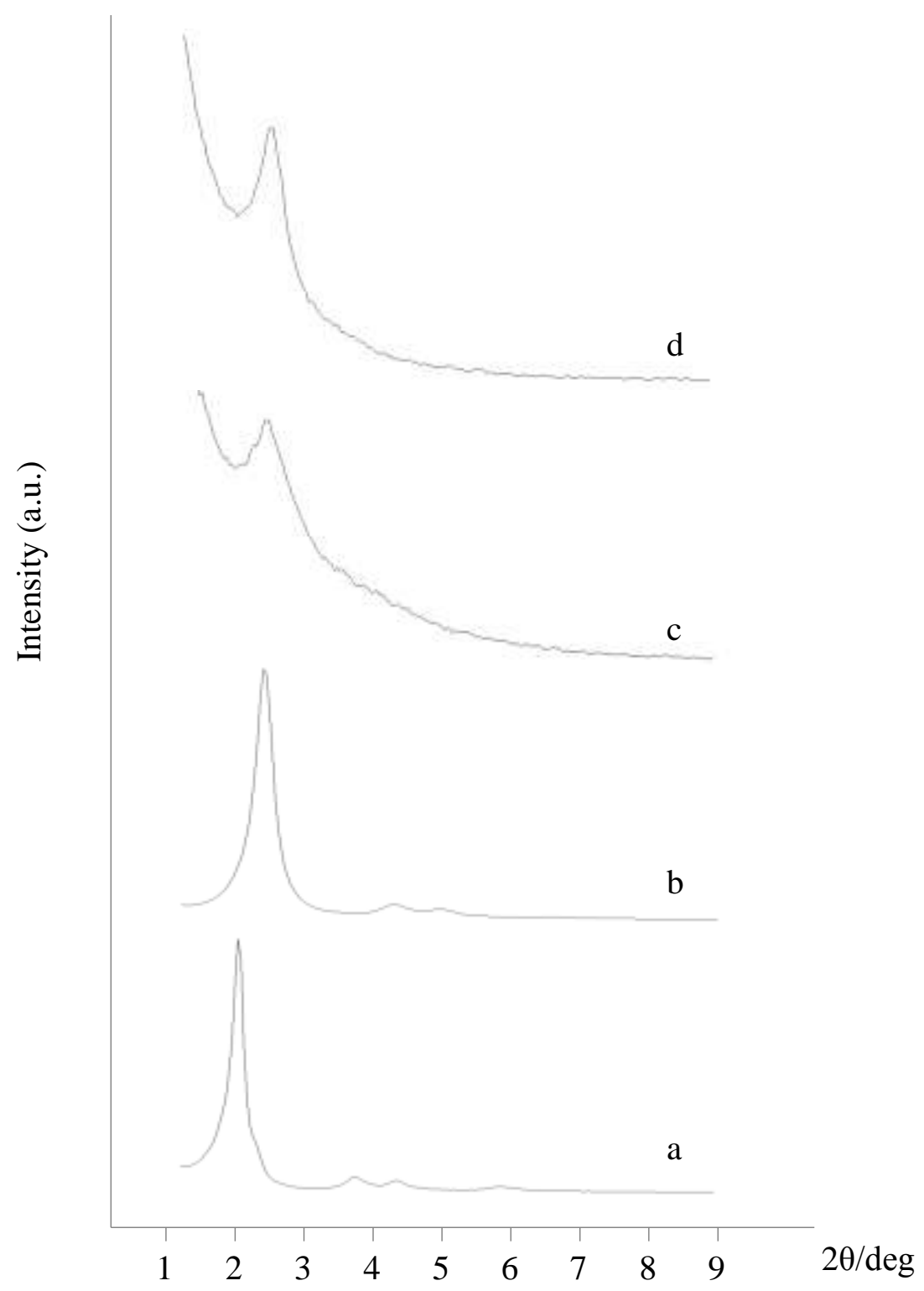

247 Figure 1. Powder X-ray patterns of the solids (a) MCM-41 as-synthesized, (b) MCM-41 calcined, (c) E-FO and (d) E-FA.

250 In addition to PXRD patterns, Fig. 2 shows FESEM and TEM images of the different bare and 251 functionalized materials. By means of FESEM observation, characterization of the shape and 252 size of the particles was performed. MCM-41 microparticles showed a size in the microscale 253 and irregular morphology. The comparison of the images before and after loading with FO and 254 FA and functionalization with N3 allowed concluding that neither loading nor functionalization significantly modified the external surface suggesting a complete encapsulation of the vitamins 
in the support. After loading with FO and FA and functionalization with polyamines, the MCMin shape, with a particle size of $708 \pm 102,779 \pm 131$ and $752 \pm 89 \mathrm{~nm}$ for bare calcined MCM-41, E-FO and E-FA, respectively. Moreover, the typical channels of the mesoporous matrix are seen as alternate black and white stripes, or as a pseudo hexagonal array of pore voids.
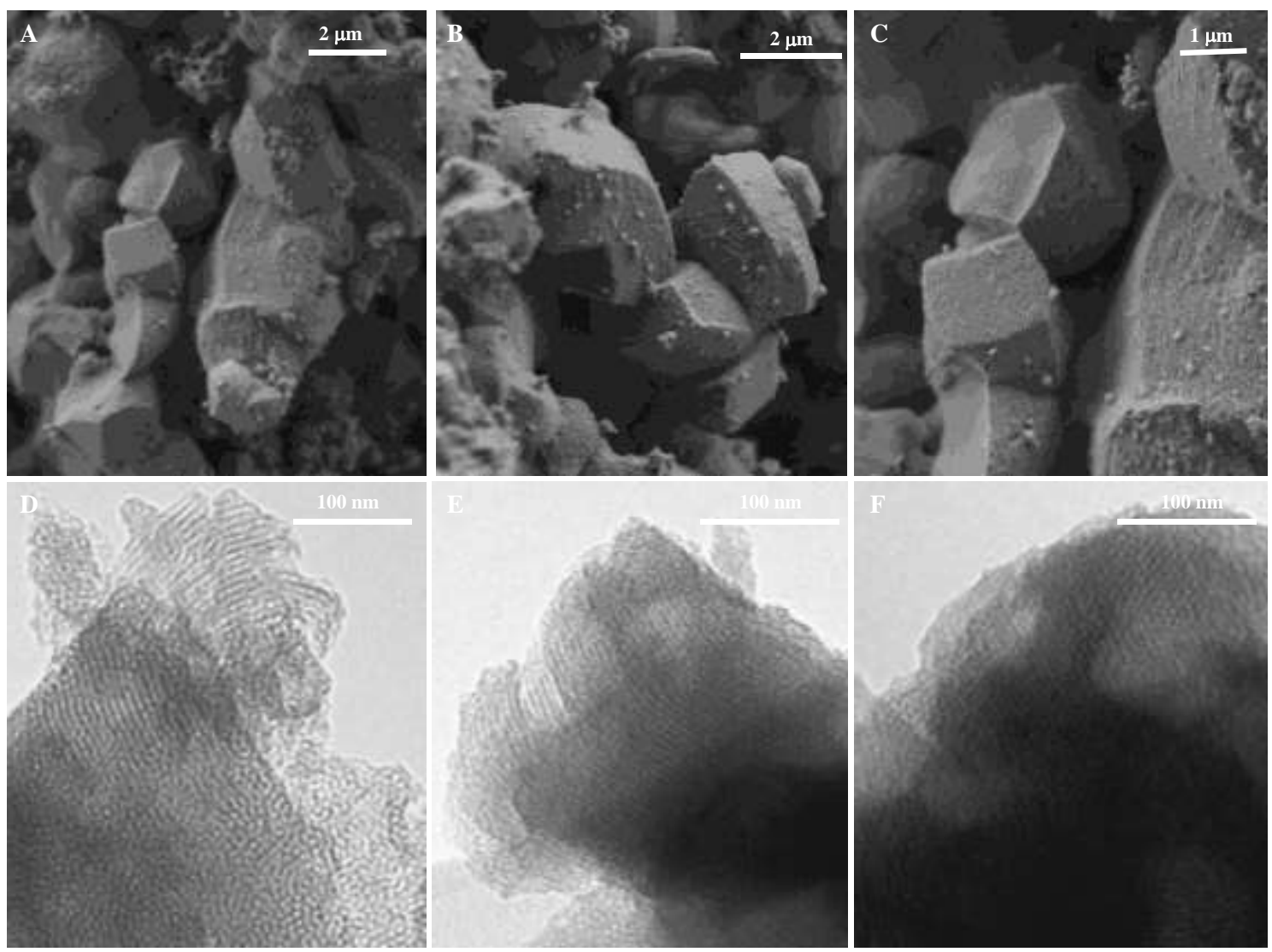

Figure 2. Characterization of particle size, particle shape and pore system by means of FESEM (A-C) and TEM (D-F). (A, D) calcined MCM-41; (B, E) E-FO and (C, F) E-FA.

The $\mathrm{N}_{2}$ adsorption-desorption isotherms of the starting MCM-41 calcined material, and of the loaded and functionalized solids, can be found in Figure 3. The MCM-41 material curve shows a well-defined adsorption step at intermediate $\mathrm{P} / \mathrm{P}_{0}$ values, which corresponds to a type IV isotherm that is typical of mesoporous materials. The isotherms of E-FO and E-FA show mesoporous system curves with partially filled mesopores. The entrapment of FA in mesopores 
271 seemed more efficient than FO, and in exactly the same way as the release studies, which

272 showed greater FA release.

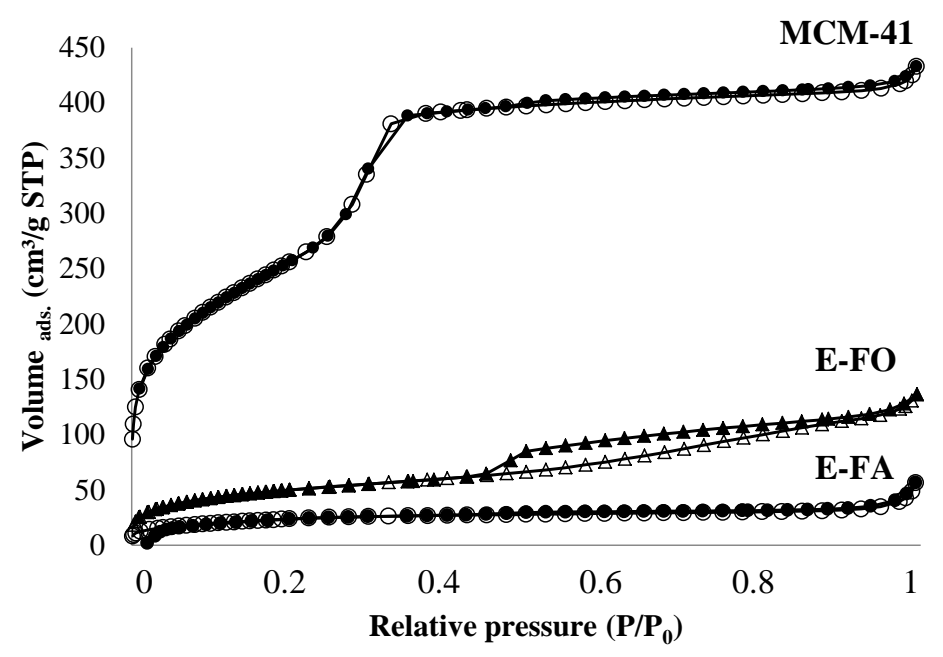

275 Figure 3. Nitrogen adsorption-desorption isotherms for MCM-41 mesoporous material, E-FO and E-FA materials.

278 Table 1 displays the change in the structural properties of the starting material after the loading 279 and functionalization processes. The values of specific surface, pore volume and pore size in $\mathbf{E}-$

280 FO and E-FA indicate significant pore blocking and the subsequent absence of appreciable 281 mesoporosity due to the incorporation of vitamins into the mesopores, as well as a reduced surface area because of the attachment of amine gates.

284 Table 1. Analytical and structural parameters from $\mathrm{N}_{2}$ adsorption-desorption isotherms.

\begin{tabular}{cccc}
\hline & SBET $\left(\mathrm{m}^{2} / \mathrm{g}\right)$ & Pore volume $\left(\mathrm{cm}^{3} / \mathrm{g}\right)$ & Pore size $(\mathrm{nm})$ \\
\hline MCM-41 & 932.61 & 0.46 & 2.72 \\
E-FA & 88.75 & 0.06 & - \\
E-FO & 183.39 & 0.19 & - \\
\hline
\end{tabular}


286 Functionalization efficiency was verified by the zeta potential determinations of bare MCM-41,

287 MCM-41 loaded with FO/FA, and MCM-41 loaded and functionalized with amines. Bare particles revealed an average negative zeta potential of $-31 \mathrm{mV}$. After loading particles with FO/FA, the zeta potential changed slightly to values of ca. $-30 \mathrm{mV}$. Yet after functionalization with $\mathrm{N} 3$, the zeta potential changed positively to values of ca. $50 \mathrm{mV}$ for E-FO and E-FA, which confirmed the attachment of amines to the particle surface.

\section{Release studies}

The release studies confirmed the mechanism of the amine-gated MSPs to modulate vitamin release according to the $\mathrm{pH}$ of the medium. The $\mathrm{pH}$-dependent releases of the encapsulated FO and FA are shown in Figure 4. Gates were largely closed at $\mathrm{pH} 2$ and the vitamin was barely detected, which confirmed that vitamin delivery was hindered by the combination of the low solubility of the vitamins under acidic conditions, the effect of the amines anchored to the surface of MSPs and the polyammonium groups-anionic species interaction. At an acidic $\mathrm{pH}$, polyamines were transformed into polyammonium groups, which adopted a rigid-like conformation due to Coulombic repulsions and coordinate anions (phosphates present in solution), which blocked pores and avoided vitamin release [8,34].

In contrast, FO and FA showed a progressive release among time at $\mathrm{pH}$ 7.5. After $2 \mathrm{~h}$, maximum vitamin releases were obtained at $\mathrm{pH} 7.5$, with $41.9 \pm 7.2 \mathrm{mg} \mathrm{FO} / \mathrm{g}$ solid for E-FO and 84.3 $\pm 7.8 \mathrm{mg} \mathrm{FA} / \mathrm{g}$ solid for E-FA. The maximum released amounts were used to calculate the equivalent amount of solids needed in the stability assays to make a comparison between the free and encapsulated FO and FA. A sustained release was produced because polyamines were less protonated at a neutral $\mathrm{pH}$, and the Coulombic repulsion between them and the affinity for anions significantly reduced. These effects, along with increased vitamin solubility, allowed the delivery of FO and FA from pores. This $\mathrm{pH}$-responsive delivery effect has been suggested to be 
311 suitable for releasing vitamins in the gastrointestinal tract (closed gates in the stomach, opened 312 gates in the intestine) [12]. Encapsulation was also expected to protect vitamins from 313 degradation after exposure to environmental agents (vide infra).
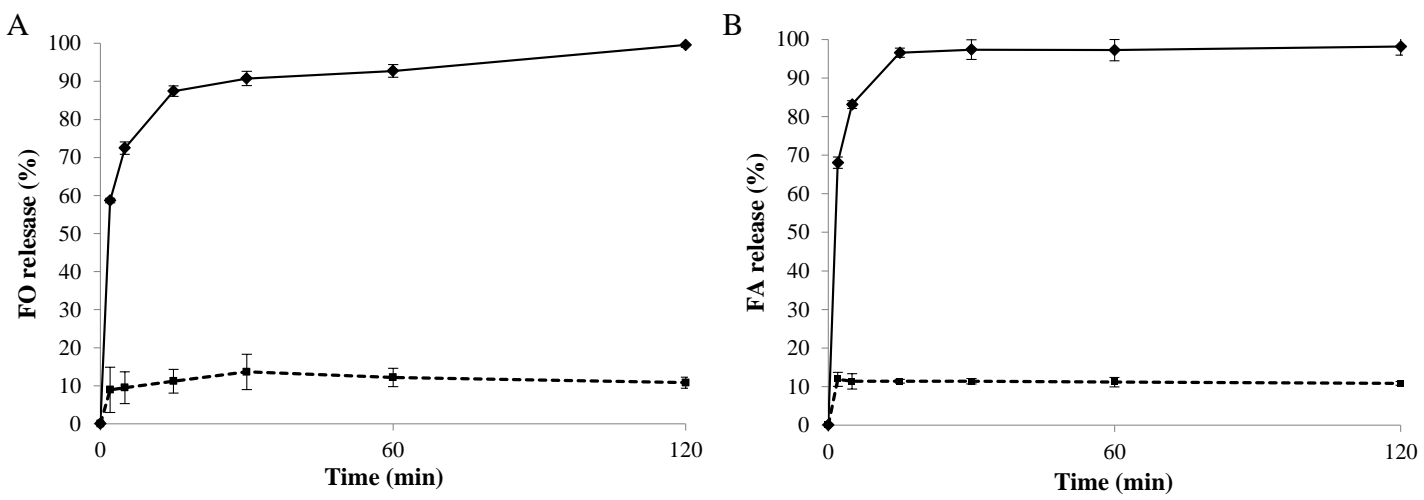

316 Figure 4. Release profiles of vitamin from the pores of E-FO (A) and E-FA (B) in PBS at pH

2.0 (dotted lines) and pH 7.5 (solid lines). Values are Means $\pm \mathrm{SD}, \mathrm{n}=3$.

\section{Stability assays}

The influence of diverse external agents related to food processing or storage, such as $\mathrm{pH}$, temperature and light, on the stability of free 5-formyltetrahydrofolate and folic acid (F-FO and F-FA) and the corresponding entrapped vitamins (E-FO and E-FA) was studied.

$\mathrm{pH}$

The study of the effect of $\mathrm{pH}$ on the stability of $\mathrm{FO}$ and $\mathrm{FA}$ at different $\mathrm{pH}$ values was conducted in two steps. In the first step, water solutions of free FA and FO were adjusted to different $\mathrm{pHs}$ and stirred for $1 \mathrm{~h}$ before being analyzed by HPLC. Figure 5 shows the detected concentrations of FA and FO (in terms of recovery) in the aqueous solutions under all the study conditions (i.e. $\mathrm{pH} 1$-10). As observed, recoveries reached values of ca. $100 \%$ from $\mathrm{pH} 5$ to 10 , which confirms the stability of both molecules at these $\mathrm{pH}$ values. Below this $\mathrm{pH}$ range, the 


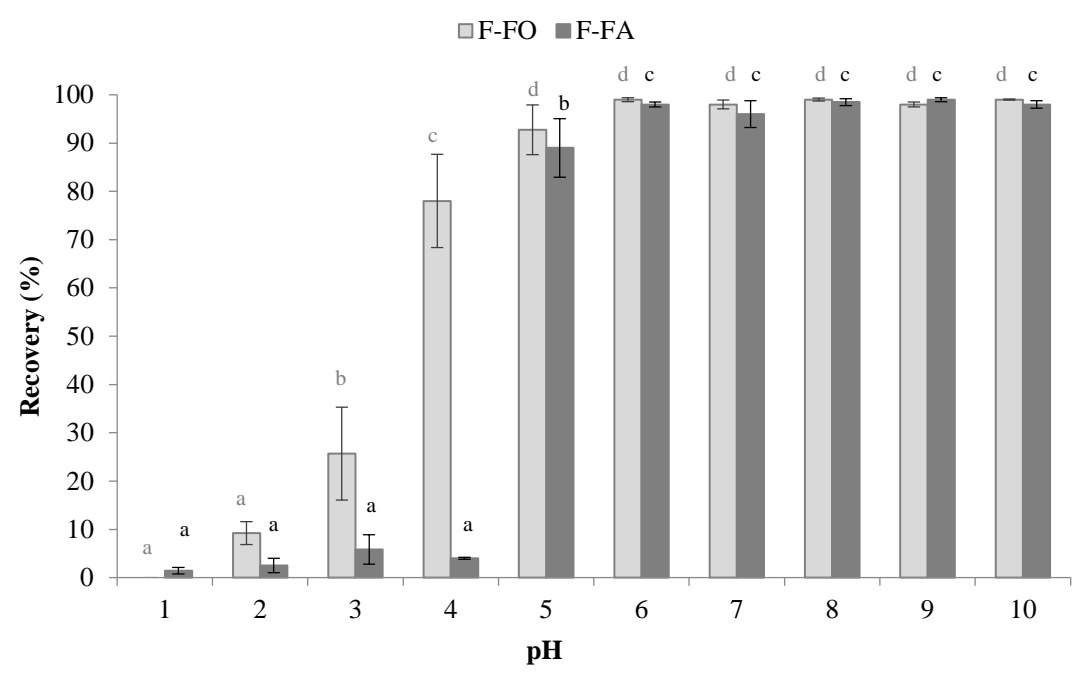

Figure 5. F-FO and F-FA recoveries at different $\mathrm{pH}$ values. Different letters in the bars indicate statistically significant differences $(\mathrm{p}<0.05)$ from levels of $\mathrm{pH}$. Values are Means $\pm \mathrm{SD}, \mathrm{n}=3$.

The drop in the recovery of FO and FA at an acidic $\mathrm{pH}$ can be explained by three phenomena: (a) loss of solubility; (b) interconversion into other derivatives; (c) oxidative degradation. Folates are slightly soluble at an acidic $\mathrm{pH}$, and are highly soluble under neutral/basic conditions due to the protonation and deprotonation of molecules in aqueous environments [32]. In addition to oxidative degradation, FO can nonenzymatically interconvert with 5,10methenyltetrahydrofolate through changes in $\mathrm{pH}$, temperature and oxygen [35]. 5,10methenyltetrahydrofolate is formed by the acidification of 5 -formyltetrahydrofolate because one molecule of water is lost (dehydration), which leads to the cyclization of the molecule in a reversible manner. The equilibrium gradually shifts toward 5,10-methenyltetrahydrofolate, and

347 its formation becomes faster the lower $\mathrm{pH}$ becomes [36].

Bearing all these factors in mind, which could explain loss of recovery at an acidic $\mathrm{pH}$, in a second step, experiments were run to determine the amount of vitamins lost at an acidic $\mathrm{pH}$. In 
them aqueous solutions of free FA and FO were adjusted to $\mathrm{pH} 2$, stirred for $1 \mathrm{~h}$ and then $\mathrm{pH}$ was adjusted to 7.5 before the HPLC analysis. The percentage of vitamins determined at $\mathrm{pH} 2$ and after neutralization to $\mathrm{pH} 7.5$ is shown in Fig. 6A, where almost no recovery of vitamins is detected after stirring them for $1 \mathrm{~h}$ at $\mathrm{pH} 2$ (which agrees with Fig. 5). However, the vitamins reappeared with a percentage of ca. $40 \%$ for F-FO and of ca. $72 \%$ for F-FA after neutralizing the $\mathrm{pH}$. F-FA gave higher values after adjusting to the neutral value than free FO, but none of them achieved complete recovery. Some studies have revealed that FA might not be soluble at a low $\mathrm{pH}$ [12], but other authors have reported its degradation at an acidic $\mathrm{pH}$ [29]. The natural folate showed less stability at an acidic $\mathrm{pH}$ than FA, probably due to degradation [36].

Similar studies to those shown above have been conducted in the presence of ascorbic acid (AA), and their results are shown in Fig. 6B. This antioxidant was included because previous studies have demonstrated that its incorporation increases folate stability as oxidation reactions are prevented [35]. As for the vitamins supplemented with AA, the F-FO concentration only slightly increased (from $40 \%$ in the absence of AA to 50\%). However, AA remarkably influenced the stability of F-FA, and revealed a recovery of almost $90 \%$.

Lastly, the effect of $\mathrm{pH}$ changes on the encapsulated vitamins was studied in order to prove the protective function of the support. As seen in Fig. 6C, minor vitamin recoveries took place at pH 2. When $\mathrm{pH}$ was adjusted to 7.5, E-FO and E-FA were almost fully detectable with a recovery of $94 \%$ and $99 \%$, respectively. Both encapsulated vitamins were highly preserved in the acidic environment by the $\mathrm{pH}$-responsive gated material. As a result, the highly protective function of the MSPs functionalized with amines at a low $\mathrm{pH}$ was evidenced by both vitamins.

371 This approach better improved the stability of vitamins than the strategy reported to enhance the 372 stability of natural folates (addition of antioxidants). Neither the free form nor the vitamins supplemented with AA were as stable at an acidic $\mathrm{pH}$ as they were inside the pores of MSPs. 

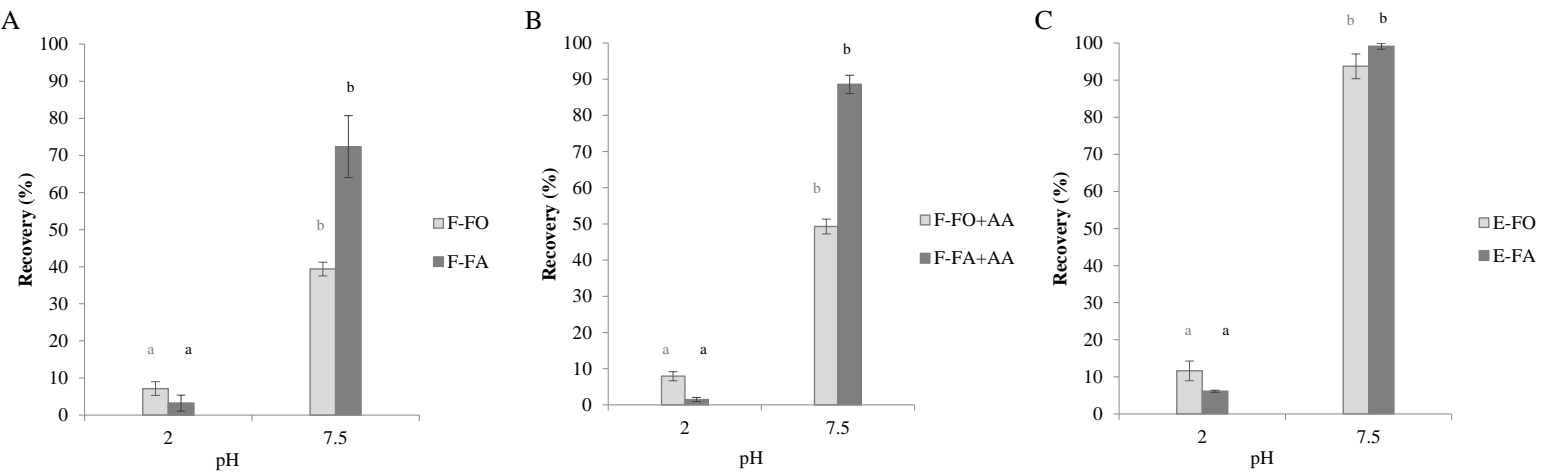

Figure 6. FO and FA recoveries after $\mathrm{pH}$ changes for free vitamins (A), free vitamins in presence of ascorbic acid (B) and encapsulated vitamins (C). Different letters in the bars indicate statistically significant differences $(\mathrm{p}<0.05)$ from levels of $\mathrm{pH}$. Values are Means $\pm \mathrm{SD}$, $\mathrm{n}=3$.

Temperature

382

Previous experiments conducted with vitamins dissolved in PBS at temperatures below $100{ }^{\circ} \mathrm{C}$ had no impact on their stability (data not shown). In order to investigate the impact of higher temperatures on the vitamins, a study was carried out by simulating sterilization conditions (121 ${ }^{\circ} \mathrm{C}, 1$ bar) at different times. The temperature assays performed in the autoclave are presented in Figure 7. The results showed that encapsulated folate did not significantly reduce vitamin content at various exposure times. In contrast, F-FO revealed a significant loss of ca. $27 \%$ after 15 min, probably due to the formation of interconversion products [30]. With FA, no significant differences were obtained for both the E-FA and F-FA results. These results are in accordance with previous studies that have reported good FA stability after thermal exposure in the solid state and with dissolution $[24,26]$. Synthetic vitamin has been suggested to be the most stable type in the folate group because of its oxidized p-teridin ring [37]. The thermostability of FA and FO has been previously reported as being similar at a neutral $\mathrm{pH}$ [23]. The results obtained with the encapsulated vitamins revealed that entrapped FO could bear up under thermal pressure exposure and greater stability after proving FO encapsulation. However, encapsulated FA could also resist the burden of thermal pressure as well as its free form. 

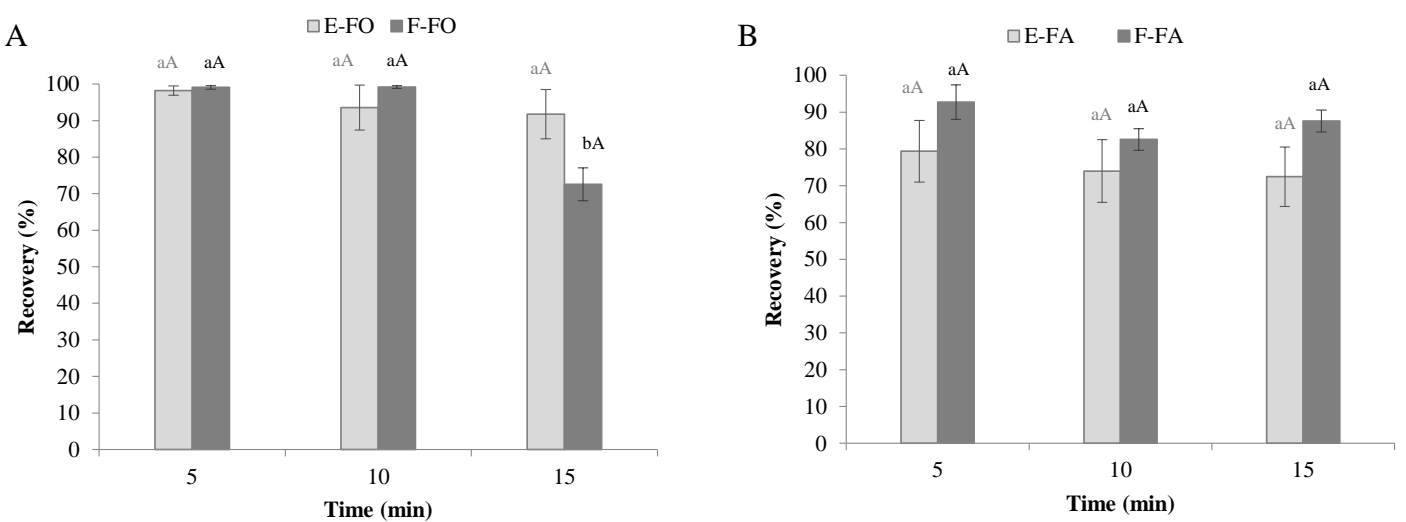

Figure 7. Influence of temperature exposure on the stability of encapsulated (E-) and free (F-)

FO (A) and FA (B) vitamins. Different letters in the bars indicate statistically significant differences $(p<0.05)$ from levels of time exposure (small letters) and differences between the encapsulated FO/FA or in their free form (capital letters). Values are Means $\pm \mathrm{SD}, \mathrm{n}=3$.

Light

Previous articles, which have reported the influence of various light sources on the degradation of synthetic FA, have investigated the impact of visible and UV light on both vitamins [25]. Preliminary experiments revealed no or very little degradation after $6 \mathrm{~h}$ (data not shown). Therefore, assays were carried out from $8 \mathrm{~h}$ to $16 \mathrm{~h}$.

Visible light assays were conducted with a lamp, which generated visible light with an intensity ca. $8 \mathrm{~mW} / \mathrm{cm}^{2}$. The results obtained from visible light experiments are presented in Figure 8. The good stability of FO (Fig. 8A) after light exposure was evidenced. Neither F-FO nor E-FO showed degradation during visible light exposure.

With FA (Fig. 8B), F-FA showed considerable loss after $8 \mathrm{~h}$ of visible light exposure, with a remaining averaged amount of $40 \%$. Gradual reduction of F-FA was detected up to $12 \mathrm{~h}$

414 irradiation, with a low value of $12 \%$, and total degradation occurred after $16 \mathrm{~h}$ with an average remaining amount of 3\%. Conversely, E-FA was well-protected by the functionalized support and a non-significant decrease was detected. 
417 Given F-FA's tendency to be degraded by light in solution, the effect of antioxidant AA was 418 also evaluated as a strategy to improve stability and to confirm the mechanism of degradation 419 (i.e. oxidation). Free FA dissolution supplemented with AA showed marginal fluctuation after $42016 \mathrm{~h}$ of visible light exposure, but appeared to stabilize FA substantially in the same way as the encapsulation system.
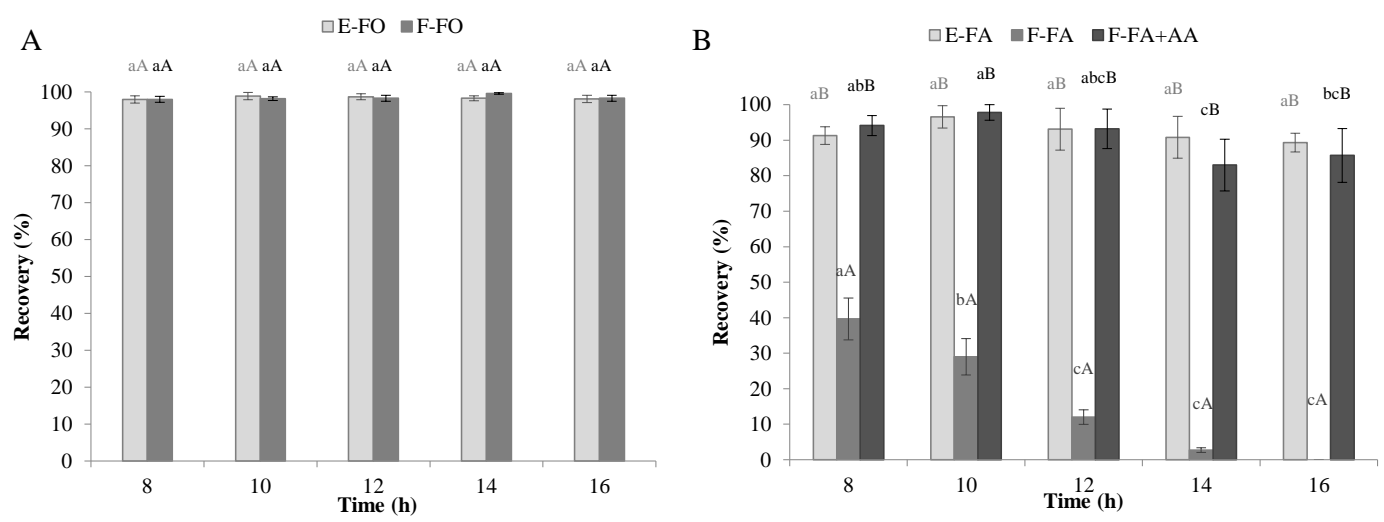

424 Figure 8. Influence of visible light exposure on the stability of encapsulated (E-) and free (F-) FO (A) and FA (B) in presence or not of ascorbic acid. Different letters in the bars indicate statistically significant differences $(\mathrm{p}<0.05)$ from levels of visible light exposure (small letters) and differences between the encapsulated FO/FA or in their free form (capital letters). Values are Means $\pm \mathrm{SD}, \mathrm{n}=3$.

Figure 9 shows the free and encapsulated FO and FA recoveries after UV light exposure with an estimated intensity of $4 \mathrm{~mW} / \mathrm{cm}^{2}$. The results revealed that E-FO and F-FO exhibited good stability under UV light (Fig. 9A). Hence natural folate was highly stable in both the free and encapsulated forms. FA stability was affected by UV light (Fig. 9B) and F-FA showed losses after $8 \mathrm{~h}$ of UV exposure. As in the visible light assays, the impact of AA enhanced the good stability of the synthetic vitamin and demonstrated more effective protection than the mesoporous system after $16 \mathrm{~h}$ of light exposure. 

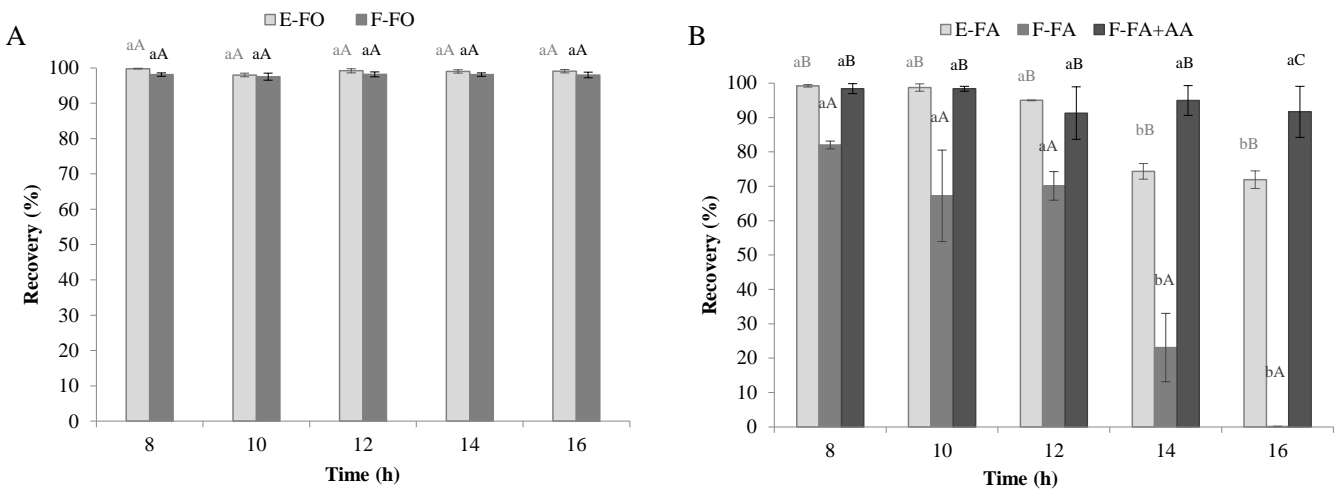

438

439

440

441

442

443

444

445

446

447

448

449

450

451

452

453

454

455

456

457

458

Figure 9. Influence of UV light exposure on the stability of encapsulated (E-) and free (F-) FO (A) and FA (B) in presence or not of ascorbic acid. Different letters in the bars indicate statistically significant differences $(\mathrm{p}<0.05)$ from levels of visible light exposure (small letters) and differences between the encapsulated FO/FA or in their free form (capital letters). Values are Means $\pm \mathrm{SD}, \mathrm{n}=3$.

Summing up the two light experiments, it was noted that FO did not degrade under either visible light exposure or UV light stress, but FA was susceptible to visible and UV light exposure. However, the mesoporous system was able to efficiently protect the vitamin and stability was greatly enhanced.

This study simulated indirect light-induced stress and, therefore, intensities until degradation occurred. We were unable to compare these results with previous studies as they all used direct exposures $[25,27,28]$. Even though it has been suggested to be considerably more stable at $\mathrm{pH}$ 7.5 than in acidic media [29], we detected complete oxidative degradation after $16 \mathrm{~h}$ of visible light and UV exposure. Akhtar et al. [25] suggested the mechanism of this oxidative degradation as they hinted at degradation being produced in the C9-N10 position. Aqueous solution can form various radiolytic products, which initiate oxidative dehydrogenation and lead to an enamine compound. This intermediate form is highly susceptible in acidic media and can undergo fast degradation. In alkaline media, this decomposition process has been proposed to take place more slowly, but it also led to irreversible cleavage between C9-N10 bonding after 16 
$\mathrm{h}$ of light exposure. This caused final decay in the separation of the p-teridin moiety from $\mathrm{p}$ aminobenzoylglutamate $[25,27]$.

461 Encapsulated FA, which was solved in PBS ( $\mathrm{pH}$ 2) in the stability assays, showed good stability. Not even the degradation-favorable acidic surrounding could hardly affect E-FA compared to the free form. Apart from successful FA improvement through encapsulation, antioxidant AA enhanced stability in the same way. Compared to the well-known protective mechanism of antioxidants [38], the protective function of the mesoporous support has not been reported to date, and the stabilizing mechanism remains unclear. Although it is fully accepted that MSPs show very little absorption within the visible and ultraviolet range [39,40], enhanced encapsulated vitamin recovery was confirmed herein. The possible role of MSPs as a stability enhancer could hinder access to weak points (C9-N10 bonding) by entrapping the vitamin in mesopores in such a way that conformational transformations of the molecule are avoided.

\section{Conclusions}

The successful entrapment of natural FO and synthetic FA in pH-responsive MSPs and the controlled release of the compounds that mimic the gastrointestinal tract were accomplished herein. The ability of MSPs to protect vitamins after environmental degradation was clearly evidenced. The stability assays revealed that encapsulated FO and FA were effectively protected against degradation at an acidic $\mathrm{pH}$ compared to their free from. The sterilization studies showed that encapsulation allowed vitamins to withstand thermal exposure and enhanced their stability. The results obtained after exposure to visible and UV light displayed good stability for free FO, which was not influenced by encapsulation, but improved FA stability after entrapment in MSPs. When considering the protective effect of MSPs against external agents and acidic stomach conditions, and progressive delivery with time under the intestinal conditions, the FOand FA-loaded supports proposed herein can be considered promising potential systems as supplements for food systems. 
Authors gratefully acknowledge the financial support from the Ministerio de Economía y

Competitividad (Projects AGL2012-39597-C02-01, AGL2012-39597-C02-02 and MAT201238429-C04-01), FEDER founding and the Generalitat Valenciana (Project PROMETEOII/2014/047). M.R.R. and E.P.E. are grateful to the Ministerio de Ciencia e Innovación for their grants (AP2010-4369 and AP2008-0620).

492

\section{References}

494

[1] Aznar, E., Oroval, M., Pascual, L., Murguía, J.R., Martínez-Máñez, R., Sancenón, F., Gated 495 materials for on-command release of guest molecules, Chem. Rev. 116 (2016) 561-718.

496

[2] Choudhari, Y., Hoefer, H., Libanati, C., Monsuur, F., McCarthy, W., Mesoporous silica

497 drug delivery systems, in: Shah, N., Sandhu, H., Choi, D.S., Chokshi, H., Malick, A.W. (Eds.), Amorphous solid dispersions, Springer, New York, USA, 2014, pp. 665-693.

499

[3] Slowing, I.I., Vivero-Escoto, J.L., Wu, C.W., Lin, V.S.Y., Mesoporous silica nanoparticles as controlled release drug delivery and gene transfection carriers, Adv. Drug Deliv. Rev. 60 501 (2008) 1278-1288.

502

[4] Popat, A., Hartono, S.B., Stahr, F., Liu, J., Qiao, S.Z., Lu, G.Q., Mesoporous silica nanoparticles for bioadsorption, enzyme immobilisation, and delivery carriers, Nanoscale 504 3(2011), 2801-2181.

505

[5] Li, Z., Barnes, J.C., Bosoy, A., Stoddart, J.F., Zink, J.I., Mesoporous silica nanoparticles in biomedical applications, Chem. Soc. Rev. 41(2012) 2590-2605.

507 [6] Pérez-Esteve, E., Oliver, L., García, L., Nieuwland, M., de Jongh, H.H., Martínez-Máñez, 508 R., Barat, J.M., Incorporation of mesoporous silica particles in gelatine gels: effect of particle 509 type and surface modification on physical properties, Langmuir 30 (2014) 6970-6979.

510 [7] Song, N., Yang, Y.W., Molecular and supramolecular switches on mesoporous silica nanoparticles, Chem. Soc. Rev. 44 (2015) 3474-3504. 
512 [8] Bernardos, A., Aznar, E., Coll, C., Martínez-Mañez, R., Barat, J.M., Marcos, M.D.,

513 Sancenón, F., Benito, A., Soto, J., Controlled Release of Vitamin B2 using mesoporous 514 materials functionalized with amine-bearing gate-like scaffoldings, J. Control. Release 131 515 (2008) 181-189.

516 [9] Clifford, N.W., Iyer, K.S., Raston, C.L., Encapsulation and controlled release of 517 nutraceuticals using mesoporous silica capsules, J. Mater. Chem. 18 (2008) 162-165.

518 [10] Kapoor, M.P., Vinu, A., Fujii, W., Kimura, T., Yang, Q., Kasama, Y., Yanagi, M, Juneja, 519 L.R., Self-assembly of mesoporous silicas hollow microspheres via food grade emulsifiers for 520 delivery systems, Micropor. Mesopor. Mat. 128 (2011) 187-193.

521 [11] Rashidi, L., Vasheghani-Farahani, E., Rostami, K., Gangi, F., Fallahpour, M., Mesoporous 522 silica nanoparticles as a nanocarrier for delivery of vitamin C. Iran. J. Biotechnol. 11 (2013) 209-213.

524 [12] Pérez-Esteve, E., Fuentes, A., Coll, C., Acosta, C., Bernardos, A., Marcos, M.D., Martínez525 Máñez, R., Barat, J.M., Modulation of folic acid bioaccesibility by encapsulation in ph526 responsive gated mesoporous silica particles, Micropor. Mesopor. Mat. 202 (2015) 124-132.

527 [13] Cotea, V.V., Luchian, C.E., Bilba, N., Niculaua, M., Mesoporous silica SBA-15, a new 528 adsorbent for bioactive polyphenols from red wine. Anal. Chim. Acta 732 (2012) 180-1855.

529 [14] Popova, M., Szegedi, A., Mavrodinova, V., Tušar, N.N., Mihály, J., Klébert, S., Benbassat, 530 N., Yoncheva, K., Preparation of resveratrol-loaded nanoporous silica materials with different 531 structures. J. Solid State Chem. 219 (2014) 37-42.

532 [15] Izquierdo-Barba, I., Vallet-Regí, M., Kupferschmidt, N., Terasaki, O., Schmidtchen, A., 533 Malmsten, M., Incorporation of antimicrobial compounds in mesoporous silica film monolith. 534 Biomaterials 30 (2009) 5729-5736.

535 [16] Bernardos, A., Marina, T., Žáček, P., Pérez-Esteve, E., Martínez-Mañez, R., Lhotka, M., 536 Kourimská, L., Pulkrávek, J., Klouček, P., Antifungal effect of essential oil components against 537 Aspergillus niger when loaded into silica mesoporous supports. J. Sci. Food Agr. (2014). 

mesoporous silica nanoparticles, Food Control 56 (2015) 77-85.

541 [18] Veith, S.R., Hughes, E., Pratsinis, S.E., Restricted diffusion and release of aroma molecules from sol-gel-made porous silica particles, J. Control. Release 99 (2004) 315-327.

543 [19] Hisamatsu, K., Shiomi, T., Matsuura, S.I., Nara, T.Y., Tsunoda, T., Mizukami, F., 544 Sakaguchi, K, $\alpha$-Amylase immobilization capacities of mesoporous silicas with different 545 morphologies and surface properties, J. Porous Mat. 19 (2012) 95-102.

546 [20] Ball, G.F.M., Vitamins in foods, analysis, bioavailability and stability, in: CRC, Boca 547 Raton, 2005.

548 [21] Gujska, E., Michalak, J., Klepacka, J., Folates stability in two types of rye breads during processing and frozen storage, Plant Food Hum. Nutr. 64 (2009) 129-134.

550 [22] Joint FAO \& WHO, FAO/WHO Expert Consultation on Human Vitamin and Mineral 551 Requirements, Food and Nutrition Division, FAO Rome, 2001, pp. 53-63.

552 [23] Indrawati, Arroqui, C., Messagie, I., Nguyen, M.T., Loey, A., Hendrickx, M., Comparative 553 study on pressure and temperature stability of 5-methyltetrahydrofolic acid in model systems and in food products, J. Agr. Food Chem. 52 (2004) 485-492.

555 [24] Vora, A., Riga, A., Dollimore, D., Alexander, K.S., Thermal stability of folic acid, 556 Thermochim. Acta 392 (2002) 209-220.

557 [25] Akhtar, M.J., Khan, M.A., Ahmad, I., Identification of photoproducts of folic acid and its 558 degradation pathways in aqueous solution, J. Pharm. Biomed. Anal. 31 (2003) 579-588.

559 [26] Nguyen, M.T., Oey, I., Verlinde, P., van Loey, A., Hendrickx, M., Model studies on the 560 stability of folic acid and 5-methyltetrahydrofolic acid degradation during thermal treatment in 561 combination with high hydrostatic pressure, J. Agr. Food Chem. 51 (2003) 3352-3357.

562 [27] Off, M.K., Steindal, A.E., Porojnicu, A.C., Juzeniene, A., Vorobey, A., Johnsson, A., 563 Moan, J., Ultraviolet photodegradation of folic acid, J. Photochem. Photobiol. B 80 (2005) 4755. 
567 [29] Yakubu, S., Muazu, J., Effects of variables on degradation of folic acid, Der Pharmacia Sinica 1 (2010) 55-58.

[30] Jastrebova, J., Axelsson, M., Strandler, H.S., Jägerstad, M., Stability of dietary 5-formyltetrahydrofolate and its determination by HPLC: a pilot study on impact of ph, temperature and antioxidants on analytical results, Eur. Food Res. Technol. 237 (2013) 747-754.

572 [31] Butz, P., Serfert, Y., Garcia, A.F., Dieterich, S., Lindauer, R., Bognar, A., Tauscher, B., Influence of high-pressure treatment at $25{ }^{\circ} \mathrm{C}$ and $80{ }^{\circ} \mathrm{C}$ on folates in orange juice and model media, J. Food Sci. 69 (2004) SNQ117-SNQ121. and 7 at temperatures $(298.15$, 303.15, and 313.15) K, J. Chem. Eng. Data 55 (2010) 39583961.

578 [33] Juzeniene, A., Tam, T.T.T., Iani, V., Moan, J., 5-methyltetrahydrofolate can be 579 photodegraded by endogenous photosensitizers, Free Radical Bio. Med. 47 (2009) 1199-1204.

580 [34] Casasús, R., Climent, E., Marcos, M.D., Martínez-Máñez, R., Sancenón, F., Soto, J., 581 Amorós, P., Cano, J., Ruiz E., Dual aperture control on pH- and anion-driven supramolecular 582 nanoscopic hybrid gate-like ensembles, J. Am. Chem. Soc. 130 (2008) 1903-1917. [35] De Brouwer, V., Zhang, G.F., Storozhenko, S., Van Der Straeten, D., Lambert, W.E., pH stability of individual folates during critical sample preparation steps in prevision of the analysis of plant folates, Phytochem. Anal. 18 (2007) 496-508.

586 [36] Jägerstad, M., Jastrebova, J., Occurrence, stability, and determination of formyl folates in 587 foods, J.Agr. Food Chem. 61 (2013) 9758-9768.

588 [37] Scott, J., Rébeillé, F., Fletcher, J., Folic acid and folates: the feasibility for nutritional enhancement in plant foods, J. Sci. Food Agr. 80 (2000) 795-824.

590 [38] Bendich, A., Machlin L.J., Scandurra O., Burton, G.W., Wayner D.D.M., The antioxidant 591 role of vitamin C, Adv. Free Radic. Biol. Med. 2 (1986) 419-444. 
592 [39] Weiping, C., Lide, Z., Synthesis and structural and optical properties of mesoporous silica 593 containing silver nanoparticles, J. Phys. Condens. Matter 9 (1997) 7257.

594 [40] Hornebecq, V., Antonietti, M., Cardinal, T., Treguer-Delapierre, M., Stable silver 595 nanoparticles immobilized in mesoporous silica, Chem. Mater. 15 (2003) 1993-199. 